\title{
SIMULATION OF THE VARIABLE MULTIFREQUENCY RADIO EMISSION AND STRUCTURE OF THE QUASAR 2145+067
}

\author{
Y.Y. KOVALEV \\ Moscow State University - Vorobjevi gori, 119899 Moscow; \\ Astro Space Center of the Lebedev Physical Institute - \\ Profsoyuznaya 84/32, 117810 Moscow, Russia
}

Here are reported new successful results of analysis for jet in the strong radial magnetic field of an active galactic nuclei, suggested in [1].

15 -year observations of the flux density of the quasar $2145+067$ at up to 10 frequencies between 0.3 and $230 \mathrm{GHz}$ from [2-4] and other are analyzed. These observations are compared with the calculations from the model, as in [5]. In general, observational curves of flux density and combined spectra versus time are in agreement with fitted model curves (see some of our results in Figure 1a-h).

The structure of this quasar, as it would be observed using a real VLBI beam, is calculated. The calculated structure (Figure 1i) is in qualitative agreement with the observed map for the used epoch from [6].

It is concluded that both the variable emission and the structure of the quasar $2145+067$ can be explained by this model. Using fitted model parameters, the luminosity distance to the source has been preliminary estimated as $1200 \mathrm{Mpc}$. On this reason, we select the source $2145+067$ as suitable for radio measurements of the Hubble constant and the deceleration parameter of the Universe by an earlier suggested method [7].

\section{References}

[1] Kardashev, N.S. (1969) Epilogue to Russian edition of Burbidge, G.R. and Burbidge, E.M. (1967) Quasars, Freeman. Mir, Moscow.

[2] Mitchell, K.J., et al. (1994) Astrophys. J. Suppl. Ser., Vol. no. 93, p. 441.

[3] Aller, H.D., et al. (1985) Astrophys. J. Suppl. Ser., Vol. no. 59, p. 513.

[4] Teräsranta, H., et al. (1992) Astron. Astrophys. Suppl. Ser., Vol. no. 84, p. 121.

[5] Kovalev, Y.Y. and Larionov, G.M. (1994) Astron. Lett., Vol. no. 20, p. 3.

[6] Wehrle, A.E., et al. (1992) Astrophys. J., Vol. no. 391, p. 589.

[7] Kovalev, Yu.A. (1994) Astron. Astrophys. Tr., Vol. no. 5, p. 67. 

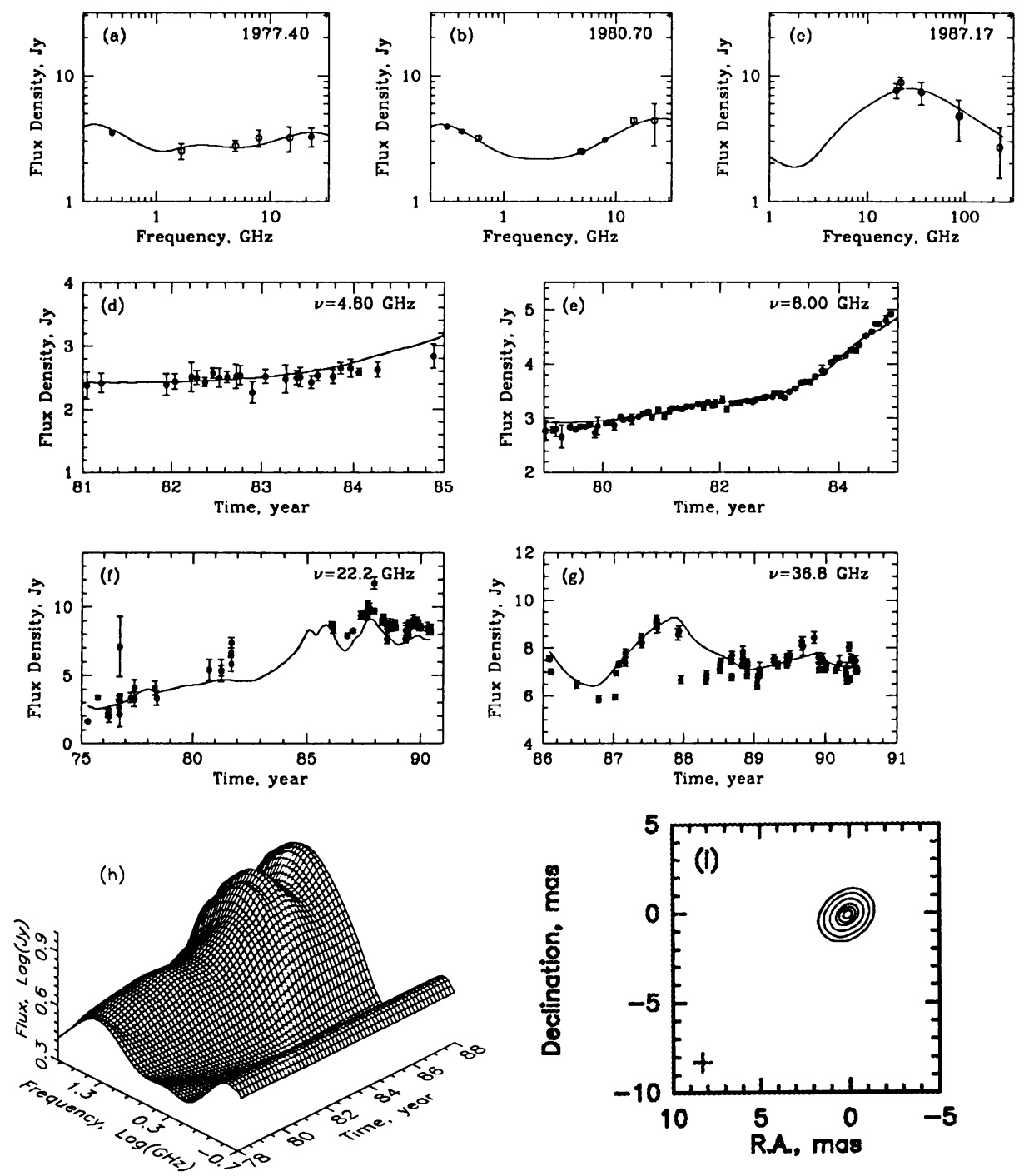

Figure 1. Some results of simulations of the variable multifrequency radio emission and structure for the quasar 2145+067. Quasi-simultaneous spectra for epochs $1977.40 \div 1987.17$ (a),(b),(c). Dots represent measured or interpolated data. Results of model calculation are shown by solid lines. The evolution of the flux density in time for the frequencies $\nu=4.8 \div 36.8 \mathrm{GHz}$ (d),(e),(f),(g), based on the observational data (dots), and model calculations (solid lines). The evolution of model spectra in time (h). Image of the jet in the model for the epoch June 6, 1986 (i) to compare with Figure $2 \mathrm{~m}$ from [6]. Contours are shown at $1,5,20,70$, and $90 \%$ of the peak brightness of $2.03 \mathrm{Jy}$. 